\title{
The Effect of Differing Intensities of Acute Cycling on Preadolescent Academic Achievement
}

\author{
Michael J. Duncan ${ }^{1}$ and Andrew J. Johnson ${ }^{2}$ \\ ${ }^{1}$ Department of Biomolecular and Sport Science, \\ Coventry University, \\ Coventry, United Kingdom
${ }^{2}$ Psychology Research Centre, Bournemouth University, Poole, United Kingdom

Running Head: Acute cycling and academic achievement

Corresponding Author: Michael Duncan

Address for Correspondence: Department of Biomolecular and Sport Science

James Starley Building

Coventry University

Email: aa8396@coventry.ac.uk 


\begin{abstract}
The present study examined the effects of differing intensity levels of acute exercise on preadolescent academic ability. In a repeated measures design, 18 preadolescent participants (mean age \pm S.D. $=9.8 \pm 1.4$ years: 9 male and 9 female) completed the Wide Range Achievement Test (WRAT 4) following 20 -minutes of rest, 20-minutes on a cycling ergometer at 50\% maximal heart rate reserve (HRR), and 20-minutes on a cycling ergometer at $75 \%$ HRR on separate days. Exercise was found to improve spelling irrespective of intensity level. Moderate levels of exercise improved reading although the effect of high levels of intensity is less clear. Both intensity levels impaired arithmetic, whilst sentence comprehension was unaffected. These findings further support the past research that indicates acute bouts of exercise can selectively improve cognition in pre-adolescent children. However, the present study finds no support for the notion that increasing the intensity of exercise accentuates benefits.
\end{abstract}

Keywords: cycling; aerobic exercise; cognitive facilitation; preadolescent children; academic achievement 


\section{INTRODUCTION}

The role of exercise on adult cognition has been extensively researched (for reviews see Lambourne \& Tomporowski, 2010; Tomporowski, 2003), with cognitive performance assessed during exercise (McMorris \& Graydon, 1996), following acute exercise (Coles \& Tomporowski, 2008; Hopkins, Davis, \& Vantieghem, 2012; Tomporowski, et al., 2005), and following long-term exercise exposure (e.g. Castelli, Hillman, Buck \& Erwin, 2007; Hopkins et al., 2012). These effects might be explained in terms of increased cerebral blood flow (Delp, et al., 2001) and heightened arousal due to exercise-induced changes in metabolic rate and reallocation of mental resources (Audiffren, 2009). In one such account (the Hypofrontality Hypothesis: Dietrich, 2003), increased arousal is conceptualised as a reallocation of less critical resources to areas of the brain employed in the coordination and execution of motor movements (e.g. prefrontal lobe). As a result of resource reallocation, cognitive processes performed within these neural regions (e.g. working memory, inhibition) exhibit a secondary benefit (Tomporowski et al., 2005; Hogervorst, Riedel, Jeukendrup \& Jolles, 1996).

There is a paucity of studies (although growing) that examine the effects of acute exercise on child cognition. This topic is pertinent for a number of reasons. First, there are concerns in respect to childhood inactivity (Riddoch, et al., 2007; see also Hillman, 2008 for contextual issues); if inactivity impairs cognition, this trend may lead to general declines in academic attainment. Second, with increased pressures on schools to achieve academic targets, the number of hours allocated to physical education have been reduced (Hardman, 2008); such reductions provide less opportunity for (acute) post-exercise enhancements of cognition. Third, it is argued that the developmental trajectory for areas of the brain that underpin cognitive control is delayed relative to other neural structures (Bunge, Dudukovic, 
Thomason, Vaidya \& Gabrieli, 2002). Since exercise is thought to enhance arousal in parts of the brain involved in cognitive control (e.g. Dietrich, 2003), one might predict that the facilitation of executive processes reported post-exercise in adults samples (Tomporowski et al., 2005; Hogervorst et al., 1996; Lichtman \& Poser, 1983) might be accentuated in children due to children exhibiting developmentally-induced executive impoverishment.

In one study, Hillman, et al. (2009) examined the effect of acute exercise on academic attainment in pre-adolescents. Children completed measures of reading, spelling, and arithmetic (using the Wide Ranging Achievement Task 3) following 20-minutes of rest or treadmill walking at $60 \% \mathrm{VO}_{2 \max }$. Testing occurred once heart rate had returned to baseline levels (within $10 \%$ of pre-exercise levels) and additionally included an electroencephalographically (EEG) monitored flanker task. Relative to rest, exercise improved reading comprehension but not spelling or arithmetic. Furthermore, reaction times for the flanker task were improved alongside improved accuracy for the incongruent trials. These effects were replicated in the electrophysiological data, wherein following exercise, increased P3 amplitudes were found for the incongruent trials. Consistent with this proposition, Drollette, Shishido, Pontifex \& Hillman (2012) found that acute bouts of treadmill walking $\left(60 \% \mathrm{VO}_{2} \max \right)$ improved performance of the flanker task but not spatial working memory, suggesting that acute exercise selectively augments inhibitory control and attention.

The findings of Hillman et al. (2009) generate a number of interesting questions. First, if acute bouts of exercise are thought to facilitate performance of tasks reliant on cognitive control, why did exercise fail to benefit arithmetic performance; a task that would necessitate working memory? Hillman et al. postulate that exercise intensity was insufficient to facilitate arithmetic, with higher 'doses' required in order to observe a benefit. One might, therefore, 
predict that a vigorous exercise intensity would lead to improvements in arithmetic. Indeed, the effects of long-term exercise exposure on academic attainment have been found in high intensity exercise only (Coe, Pivarnik, Womack, Reeves \& Malina, 2006). Alternatively, Hillman et al.'s null effect on arithmetic may simply reflect an absence of post-exercise working memory facilitation (Drolette et al., 2012), with effects of acute exercise confined to attention and inhibitory control.

Second, if increased arousal post-exercise is the mechanism implicated in cognitive facilitation, does an optimum level of post-exercise arousal exist or would cognition continue to improve with increased intensity. Initial accounts of exercise and arousal predicted an inverted U-shaped relationship (McMorris \& Graydon, 2000; see also Yerkes \& Dodson, 1908) such that moderate levels of exercise increased arousal and facilitated cognition, but when arousal approaches maximal levels performance begins to deteriorate (Levitt \& Gutin, 1971; Brisswalter, Durand, Delignieres \& Legros, 1995). Prior adult studies examining the effect of exercise induced fatigue on cognitive variables have evidenced an inverted $U$ effect of increasing exercise intensity on coincidence anticipation (Lyons, Al-Nakeeb \& Nevill, 2008) and reaction times (Chmura, Nazar \& Kaciuba-Uscilko, 1994). Indeed, recent metaanalysis has concluded that acute moderate intensity exercise has a positive effect on working memory tasks (McMorris, Sproule, Turner, \& Hale, 2011) supporting the inverted U relationship. However, maximal levels of exercise intensity are not uniformly associated with declines in cognition (see Tomporowski, 2003, for review) nor can heart rate elevations uniquely explain post-exercise enhancement in concentration and attention (Budde, VoelckerRehage, Pietraßyk-Kendziorra, Ribeiro \& Tidow, 2008). Furthermore, meta-analytical data by Chang, Gapin and Etnier (2012) concluded that exercise intensities above 'light' were 
associated with enhanced cognitive performance providing the exercise protocol lasted at least 20minutes or longer (Chang et al., 2012).

More recently, work by Best (2012) examined the effects of 'exergaming' on executive function in 33,6 to 10 year old children. In their study children engaged in conditions of low and high physical activity with corresponding levels of low and high cognitive engagement for a 10-minute period. On each occasion executive function was assessed on cessation of the exercise intervention using a modified flanker task. Their results showed that physical activity, in the form of exergaming, enhanced children's speed to resolve interference from conflicting visual stimuli. The authors suggested that their findings evidenced compelling evidence that physical activity primes executive functioning in order to exert control of children's cognition and behaviour (Best, 2012), and further suggested that physical activity may be a useful addition to the school classroom to improve children's performance.

It is of interest to note that the majority of pediatric studies on this topic have examined the effect of an acute bout of exercise on cognitive variables as compared to a seated rest condition. The exercise condition in these studies has tended to be moderate in nature (e.g., 60\% $\mathrm{VO}_{2}$ max, Drolette et al., 2012; Hillman et al., 2009). Given the literature documenting a dose-response relationship between exercise intensity and health benefit in children (Parikh \& Stratton, 2011) it is perhaps surprising that studies have not yet examined whether exercise intensity plays a role in any exercise-induced effects on children's cognitive performance. Understanding the role of exercise intensity in augmenting academic and/or cognitive performance in children is an important first step in targeting interventions to enhance academic achievement whilst also enhancing health (Kristjansson, Sigfúsdóttir, Allegrante \& Helgason, 2008). As recommendations for physical activity in children stress 
the need for moderate and vigorous physical activity (Strong, et al., 2005), examining whether intensity of acute exercise (moderate versus vigorous) affects the exercise-cognition relationship would seem logical in further understanding how acute aerobic exercise may benefit cognitive performance in children.

The third point of interest concerns exercise type. Hillman et al. (2009) reported beneficial effects following 20-minutes of treadmill exercise; this is despite a meta-analysis conducted by Lambourne and Tomporowski (2010) showing that cycling produced stronger effect sizes than treadmill running. Lambourne and Tomporowski (2010, p.18) argued that cycling uses less metabolic energy compared to running and that running resulted in greater "neural interference" (presumably due to greater coordinative demands). It is, therefore, an empirically testable question whether use of a cycling ergometer produces the same (or even accentuated benefits) in academic performance.

The present study will attempt to address some of the issues raised following the Hillman et al. (2009) paper by examining the effects of different exercise intensities on preadolescent academic performance. Children will complete the WRAT 4 (a standardised measure of academic ability that assesses arithmetic, spelling, reading, and sentence comprehension) following 20-minutes of rest, exercise at 50\% maximal heart rate reserve, and exercise at $75 \%$ maximal heart rate reserve. Exercise will be conducted on a cycling ergometer in order to maximise any facilitative effect sizes (Lambourne \& Tomporowski, 2010). The study will seek to replicate the post-exercise improvements reported by Hillman et al. (2009) but further examine whether following vigorous exercise intensity effects on reading are accentuated and benefits to spelling and arithmetic develop.

\section{METHOD}




\section{Participants}

Eighteen preadolescent children (9 boys, 9 girls) aged 8-11 years (mean age \pm S.D. $=9.8 \pm$ 1.4years) from the city of Coventry, UK, participated in the study following institutional ethics approval and informed parent and child consent. Prior to testing, legal guardians completed a health history and demographics questionnaire, reported that their child was free of neurological disease, attentional disorders and physical disabilities, and indicated normal or corrected-normal vision based on a minimal 20/20 standard. A pre-exercise Physical Activity Readiness Questionnaire (PAR-Q) was also used confirm that the children did not have any condition that would be exacerbated by physical exercise. None of the children had a recognised special educational need (e.g., dyslexia), behavioral problems, and nor were they classified as gifted and talented according to school records. Children were all drawn from one school representing an area in the mid-range of socio-economic status within the city. The City Council's Education Department assisted in identifying a school within the mid range of socio-economic status to approach to participate in the first instance. Children were not given any inducement to participate and were recruited following a presentation given by the researchers to children and parents attending the school concerned.

Physical maturation was assessed by predicting the age at peak height velocity (APHV). This is a method based on the growth patterns of the upper body and legs of every individual and is compared to the average population with the aim to classify children between early, average, and late maturers. Previous studies have commonly used Tanner's scale in which children are assessed by the stage of development of their secondary sex characteristics (Tanner, 1962). However, this technique of measuring APHV was selected as non-invasive, ethically more appropriate, and more acceptable to parents and children as a gauge of maturation (Mirwald, Baxter-Jones, Bailey \& Beunen, 2002) compared to other 
methods. This confirmed that all children in this sample were prepubescent (equivalent to Tanner stage II) at the item of testing with the exception of 2 girls who were classified as Tanner stage III.

\section{Procedures}

This study employed a within subjects design whereby participants visited the laboratory on four occasions in which during those days they had not participated in physical education or other structured physical activity. All visits occurred at the same time of day. The initial session was used to decipher maximal heart rate reserve. The subsequent experimental sessions comprised a rest condition, a moderate and a vigorous intensity condition. The order of these six experimental conditions was randomised such that between two and four participants were assigned at random to one of the possible condition orders. In the first session, participants had their height $(\mathrm{cm})$ and body mass $(\mathrm{kg})$ assessed using a Seca Stadiometre and weighing scales (Seca Instruments, Frankfurt, Germany) and were fitted with a heart rate monitor (RS400, Polar Electro, Kuopio, Finland). Resting heart rate was recorded for 5 minutes in a supine position following procedures previously described (Ridgers, Stratton, Clark, Fairclough \& Richardson, 2006). This data was used to determine exercise intensities of $50 \%$ (moderate) and $75 \%$ (vigorous) of maximal heart rate reserve (Karvonen \& Vuorimaa, 1988) to be used in subsequent experimental sessions. Heart rate reserve (HRR) values of 50 (HRR50) and 75 (HRR75) per cent were used as threshold values to represent moderate and vigorous physical activity, respectively. HRR50 equates to a brisk walk (Armstrong \& Welsman, 1997; Ridgers et al., 2006), and HRR75 equates to a measure of vigorous physical activity because it is thought that this intensity increases cardiorespiratory fitness in children (Payne \& Morrow, 1993; Praikh \& Stratton, 2011). 
Following this, participants undertook 3 experimental conditions: rest, moderate intensity exercise, and vigorous intensity exercise (one condition per day/visit). Conditions were separated by 7 days. The rest session consisted of 20 minutes seated rest and the moderate and vigorous intensity exercise sessions consisted of 20minutes of aerobic exercise on a cycle ergometer (Monark Exercise $\mathrm{AB}$, Sweden) at $50 \%$ and $75 \%$ of maximal heart rate reserve for moderate and vigorous conditions respectively. This duration was chosen as it complies with the only other study that has examined the acute effect of exercise on children's academic achievement (Hillman et al., 2009). Furthermore, in the aforementioned study by Hillman et al. (2009), children exercised at an intensity of $60 \%$ of maximal heart rate. This threshold was not justified by the authors. Recognising that the use of maximal heart rate as an indicator of exercise intensity fails to account for individual differences in fitness level, maximal heart rate reserve was employed in the present study. Moreover, intensities of $50 \%$ and $75 \%$ heart rate reserve were chosen as these intensities directly relate to moderate to vigorous physical activity, the recommended intensity of physical activity for health benefit in children (Ridgers et al., 2006).

On completion of each experimental session, and once participants' HR had returned to within $10 \%$ of their pre-exercise levels, the Wide Ranging Achievement Task (WRAT4: Wilkinson and Robertson, 2006) was administered. WRAT 4 is the updated version of the academic achievement test employed by Hillman et al. (2009) and in addition to measures of word reading, spelling, and arithmetic, now included an assessment of sentence comprehension. Word reading is a measure of the number of words correctly pronounced aloud and spelling is a measure of the number of words correctly spelt. The reading test comprises 55 words ranging from three letter words such as 'see' and 'red', to more complex words such as 'ubiquitous' and 'regicidal' with children asked to read these aloud to the 
tester. In the spelling test, children are read a series of 42 words in isolation and then in context (e.g., 'go. The children want to go home') and are asked to spell these words in written form. The words range from two letter (e.g. 'go') to 9 letter (e.g. 'assiduous') words. The arithmetic score is a measure of the number of mathematical problems correctly solved. In the assessment of sentence comprehension children are asked to provide the missing word in a given sentence e.g. 'Dee is having a birthday party for her brother. He will be seven old.' or 'Although the humpback is one of the most studied of the great whales, about its social behavior remain unanswered' to measure the ability to comprehend ideas and information contained in a sentence. This paper and pencil assessment was administrated individually in a quiet room and lasted approximately 20 minutes. The WRAT was administered in the order prescribed by the test guidelines, i.e. (1) word and letter reading, (2) sentence comprehension, (3) spelling, and (4) math computation. Following the final session, participants were debriefed.

\section{RESULTS}

For the purpose of analysis standardised scores on WRAT 4 were employed. Data was screened for normality (Shapiro-Wilk, $p>.05$ ) and met the assumption.

\section{Effects of Moderate Exercise Compared to Rest}

One of the participants was unable to obtain $50 \%$ maximal heart rate reserve during the exercise intervention and was excluded from the analysis. The analysis was therefore conducted on a sample of 17 children. Figure 1 demonstrates the mean standardised scores across the moderate exercise and rest conditions for the four academic measures. A repeated measures MANOVA was conducted, wherein the independent variable was invention 
(moderate exercise or rest) and the dependent variables were standardised WRAT scores for mathematics, reading, spelling, and sentence comprehension. There was a significant multivariate effect for the intervention condition, $F(4,13)=8.73, p=.001$, Wilks' Lambda $=$ $.27, \eta_{p}{ }^{2}=.73$. Analysis of each individual dependent variable (with Bonferroni correction: $\alpha=.012$ ) was conducted. Standardised scores were significantly higher following moderate exercise for both spelling $\left(F(1,16)=8.51, p=.01, \eta_{p}{ }^{2}=.35\right)$ and reading $(F(1,16)=8.51, p=.01$, $\left.\eta_{p}{ }^{2}=.35\right)$. In contrast, standardised mathematics scores were significantly lower following moderate exercise compared to rest, $F(1,16)=25.24, p<.001, \eta_{p}{ }^{2}=.61$. There was no difference between moderate exercise and rest for sentence comprehension, $F(1,16)=.07$, $p=.80, \eta_{p}^{2}=.004$

\section{Effects of Exercise Intensity}

Six of the participants were unable to obtain $75 \%$ maximal heart rate reserve during the exercise intervention and were excluded from the analysis. The analysis was therefore conducted on a sample of 12 children. Figure 2 demonstrates the mean standardised academic performance scores across the resting, moderate exercise, and vigorous exercise conditions. A repeated measures MANOVA was conducted, wherein the independent variable was invention (resting, moderate exercise, vigorous exercise) and the dependent variables were standardised WRAT scores for mathematics, reading, spelling, and sentence comprehension. There was a significant multivariate effect for the intervention condition, $F(4,8)=7.60, p=.03$, Wilks' Lambda $=.06, \eta_{p}^{2}=.94$. Analysis of each individual dependent variable (with Bonferroni correction: $\alpha=.012$ ) was conducted. An effect of intervention was found for spelling, $F(2,22)=12.99, p<.001, \eta_{p}{ }^{2}=.54$. Further analysis of the main effect (Bonferonnicorrected comparisons: $\alpha=.016$ ) revealed that both moderate and vigorous exercise produced 
significantly higher scores than the resting condition. There was no significant difference between moderate and vigorous exercise. An effect of intervention was found for arithmetic, $F(2,22)=6.29, p=.007, \eta_{p}{ }^{2}=.36$. Further analysis of the main effect (Bonferonni-corrected comparisons: $\alpha=.016$ ) revealed that the resting condition had significantly higher score than both the moderate and vigorous exercise conditions. There was no significant difference between moderate and vigorous exercise. The effect of intervention for reading approached significance, $F(2,22)=2.64, p=.09, \eta_{p}{ }^{2}=.19$. There was no main effect of intervention for sentence comprehension, $F(1.31,14.37)=.21, p=.72, \eta_{p}^{2}=.02$.

\section{DISCUSSION}

The present study investigated the effect of an acute bout of exercise (20-minutes of ergometer cycling) at differing levels of intensity (50\% and $75 \%$ of heart rate reserve) on academic performance (WRAT 4). In the first part of the analysis $(n=17)$, relative to a 20minute resting control, exercise (at 50\% HRR) was found to both improve spelling and reading but impair arithmetic. When comparing across different exercise intensities $(n=12)$, exercise, relative to the testing control, was found to improve spelling and impair arithmetic. Neither of these effects was augmented by exercise intensity. No effects were found in respect to reading or sentence comprehension.

The findings are generally consistent with the work of Hillman et al. (2009) such that benefits at lower exercise intensities were found with spelling. Both studies failed to report any benefit of exercise on arithmetic; indeed, the present study reported that exercise (at both intensity levels) significantly impaired arithmetic. Our data therefore indicate that the absence

of arithmetic facilitation reported by Hillman et al. was not due to insufficiently high 
intensity levels of exercise. Indeed, arithmetic remained unchanged following the increase in intensity from $50 \%$ HRR to $75 \%$ HRR (mean standardised arithmetic scores for $50 \%$ and $75 \%$ HRR $=88.75$ and 88.83 , respectively); specifically, exercise impaired arithmetic for both intensities. Since completion of the mathematical problems may have necessitated the mental manipulation of figures, the null effects on arithmetic are consistent with Drolette et al. (2012) who found that acute bouts of exercise did not facilitate working memory operation. Furthermore, the findings of the current research are also broadly in agreement with those recently reported by Best (2012), who showed improvements in children's executive function following an acute 10 minute bout of combined physical activity and videogaming. Prior authors have suggested that exercise induced arousal may be the mechanism which explains enhanced cognitive performance following exercise (McMorris et al., 2011). However, if this was the case then it may be reasonable to expect that arousal would influence all aspects of WRAT4 performance. Likewise, the increased levels of brain derived neurotrophic factor found with increasing exercise intensity have also been cited as a mechanism for improvements in cognition as a result of exercise (Chang et al., 2012). However, this variable was not assessed in the present study so no claim can be made regarding this potential mechanism.

The absence of intensity effects contradicts predictions both based upon the inverted U-arousal theory (Yerkes \& Dodson, 1908) and positive linearity between exercise and cognition. One might posit, therefore, that ceiling effects are reached in respect to the effects of post-exercise facilitation on academic achievement. Further work is required to establish the most efficient exercise intensity that would improve child academic performance. It may also be that the duration of exercise was not sufficient to elicit clear benefits in cognitive performance in the present study. Twenty minutes has been cited as the minimum exercise 
duration to evidence beneficial effects on cognitive variables (McMorris et al., 2011) and in pediatric samples a longer duration of exercise may be needed to realise any benefit. There is current debate regarding the recommended level of physical activity needed for health (Praikh \& Stratton, 2011; Strong et al., 2005) with authors asserting that vigorous intensity physical activity/exercise results in the greatest health benefit in children (Praikh \& Stratton, 2011). Therefore, providing robust guidelines as to the effect of exercise intensity on academic performance in children specifically, and cognition in general, would be useful in structuring exercise recommendations for benefit of the child as a whole.

Another important distinction between the present data and that of Hillman et al. (2009) concerns the differential effects of moderate exercise on reading. Hillman et al. failed to report any benefit of exercise on reading; in contrast, the present study reported an improvement in reading following the moderate intensity exercise (50\% HRR: $n=17)$. This disparity may be explained via the different modes of exercise employed across the two studies, since Lambourne and Tomporowski (2010) highlight that bigger effect sizes are reported for cycling (as used in the present study), compared to treadmill running (as used in Hillman et al., 2009). This suggests that a more effective intervention involves a cycling ergometer, with running producing greater levels of "neural interference" (Lambourne \& Tomporowski, 2010, p.18; although, in contrast, Budde et al. 2008, proposed that exercise requiring more coordinative movements facilitated attention and concentration to a greater extent that more automated exercise). It is conceded that due to financial constraints the presence of a sufficient number of cycling ergometers may inhibit such an intervention at school-level. However, we argue that it is important to establish the presence of the any benefit under conditions optimal for the detection of the effect (i.e. with cycling, Lambourne \& Tomporowski, 2010), prior to more ecologically valid interventions. It should also be 
noted that although comparison of reading abilities between resting, 50\% HRR, and 75\% HRR was not significant $(p=.09)$, visual inspection of Figure 2 reveals a trend towards improvement for moderate exercise only. Post-hoc power analysis reveals that the study was not under-powered (strong effect size: $\eta_{p}{ }^{2}=.19$ ), with the failure to obtain significance possibly underpinned via large between-participant variability in reading. Indeed, the error sums of squares were much higher for reading compared to both spelling and arithmetic. It is therefore possible that more controls were needed in reading ability across our participants. Nevertheless, the trend merits further examination to ascertain whether facilitation in reading is confined only to moderate levels of exercise. It is also worthy to note that these results are based on a relatively small sample of children and further research would be useful that employs larger participant numbers. Furthermore, the fact that a number of children could not complete the full 20minutes of vigorous intensity exercise merits further scrutiny. Although constant vigorous intensity exercise such as that undertaken in the present study is not consistent with the normal patterns of physical activity in children (Armstrong \& Welsman, 1997), it may be that those who could not complete the vigorous intensity exercise were low fit. Fitness was not assessed in the present study and future research may wish to consider fitness as a mediating factor on the impact of exercise on cognitive performance in children. Indeed, whilst a sustained period of exercise enables greater experimental control (and is consistent with exercise and cognition manipulations used previously in the literature, e.g. Hillman et al., 2009; Tomporowski et al., 2005), it should be noted that 20-minutes of sustained aerobic exercise lacks ecological validity for such a young sample where intermittent physical activity bouts are more indicative of typical children's activity behaviour The choice to employ a sustained bout of 20-minutes moderate and vigorous exercise was however employed to ensure consistency with prior studies on this topic (Hillman et al., 2009; Tomporowski et al., 2005) and is also congruent with guidelines for 
children's health enhancing physical activity (WHO, 2011) and for the enhancement of aerobic health in children and adolescents (US Department of Health and Human Services, 2012) where a period of 20minutes sustained vigorous activity is identified. Presently, exercise interventions with children are comparably structured to that of adult samples in order to assess if similar effects are found across the lifespan. However, other studies are now needed which employ intermittent exercise interventions; such a manipulation would provide a more direct analogue to school recess exercise and enable researchers to, potentially, make a stronger case for classroom breaks. However, one should note the possible experimental confounds of such an approach, wherein rest intervals between intermittent play may produce large (fitness-based) variability in cardiovascular recovery. Finally, it may have been useful to have also assessed other relevant factors such as cognitive function and event related potentials alongside the assessment of academic achievement.

A further distinction with the Hillman et al. (2009) design concerns the incomplete counterbalancing of order conditions. There were six possible orders for the rest, $50 \%$ HRR, and $75 \%$ HRR conditions. In the present study between 2 and 4 participants were randomly assigned to those six orders. It is possible that the effects in the present study were a result of imperfect counterbalancing (particularly in respect to the novel detrimental effect on arithmetic). We argue against this interpretation for three reasons. First, in their study Hillman et al. (2009) reported no order effects following completion of the WRAT under rest and exercise conditions, suggesting that this paradigm is not susceptible to asymmetric order effects. Second, it is not clear why the imperfect counterbalancing should impair arithmetic but not spelling, reading, and comprehension. Third, the negative effect sizes on arithmetic were strong for both 50\% HRR and the combined $50 \%$ and $75 \%$ HRR analysis $\left(\eta_{\mathrm{p}}{ }^{2}=.61\right.$ and .36 , respectively). In the combined analysis means were consistently lower for both exercise 
conditions relative to rest (for standardised arithmetic: rest $=96.00,50 \% \mathrm{HRR}=88.75$, and $75 \%$ HRR $=88.83)$ : it seems improbable that the imperfect counterbalancing should impair both the exercise conditions but not the rest condition.

It is, however, worth noting that when comparing the results of the present study and that of Hillman et al. (2009), the two methods employed to determine 'moderate' exercise intensity appear to differ, with the present study employing the heart rate reserve method and the work of Hillman et al. (2009) using a \% of heart rate maximum. Notwithstanding, this apparent discrepancy, the two 'moderate' intensities are comparable (Janot, 2005). For exercise prescription, 'moderate' intensity exercise has been recommended to lie within 55$65 \%$ of HR max and 50\% of HRR (Janot, 2005). This would suggest that the differing effects between Hillman et al. and the present study at moderate exercise intensity cannot be explained via differences in exercise intensity levels.

The present study employed an updated version of WRAT that now included a score for sentence comprehension. This was unaffected by exercise (irrespective of intensity). It is not clear why exercise should have differential effects on the four measures of academic performance. Coles and Tomporowski (2008) provided some distinction in respect to the effects of acute bouts of exercise on cognition and proposed that exercise does not influence working memory processes that involve organisation and sequencing of mental operations but can facilitate the consolidation of delayed long-term memories. This distinction is, however, unable to account for the present data as memory for letter positions and wordphoneme associations is already encoded pre-study.

In summary the present study provides no strong evidence that exercise intensity moderates the improvement in pre-adolescent post-exercise academic ability. Exercise was found to improve spelling independent of intensity. Exercise improved reading, with the 
relationship with exercise intensity requiring further investigation. Exercise did not improve, but impaired, arithmetic.

\section{ACKNOWLEDGEMENTS}

The authors would like to thank Matthew Hill, Emma Eyre, Marta Campolier, and Elizabeth Bryant for their assistance in the collection of data

\section{REFERENCES}

Armstrong, N., \& Welsman, J. (1997). Young People and Physical Activity. Oxford, UK: Oxford University Press.

Audiffren, M. (2009). Acute exercise and physiological functions: a cognitive-energetics approach. In: McMorris, T., \& Tomporowski, P.D. (Eds). Exercise and cognitive function. John Wiley \& Sons. Hoboken NJ., p. 3-39.

Best, J. R. (2012). Exergaming immediately enhances children's executive function. Developmental Psychology, 48, 1501-1510.

Brisswalter, J., Durand, M., Delignières, D., \& Legros, P. (1995). Optimal and non-optimal demand in a dual task of pedalling and single reaction time: Effects on energy expenditure and cognitive performance. Journal of Human Movement Studies, 29, 1534.

Budde, H., Voelcker-Rehage, C., Pietraßyk-Kendziorra, S., Ribeiro, P., \& Tidow, G. (2008). Acute coordinative exercise improves attentional performance in adolescents. Neuroscience Letters, 441, 219-223. 
Bunge, S.A., Dudukovic, N.M., Thomason, M.E., Vaidya, C.J., \& Gabrieli, J.D.E. (2002). Immature frontal lobe contributions to cognitive control in children: evidence from fMRI. Neuron, 33, 301-311.

Castelli, D., Hillman, C., Buck, S., \& Erwin, H. (2007). Physical fitness and academic achievement in 3rd and 5th grade students. Journal of Sport and Exercise Psychology, 29, 239-252.

Chang, Y.K., Labban, J.D., Gapin, J.I., \& Etnier, J.L (2012). The effects of acute exercise on cognitive performance: A meta-analysis. Brain Research, 1453, 87-101.

Chmura, J., Nazar, K., \& Kaciuba-Uscilko, H. (1994). Choice reaction time during graded exercise in relation to blood lactate and plasma catecholamine thresholds. International Journal of Sports Medicine, 15, 172-176.

Coe, D. Pivarnik, J., Womack, C., Reeves, M., \& Malina, R. (2006). Effect of physical activity levels on academic achievement in children. Medicine and Science in Sports and Exercise, 38, 1515-1519.

Coles, K., \& Tomporowski, P.D., (2008). Effects of acute exercise on executive processing, short-term and long-term memory. Journal of Sports Science, 26(3), 333-344.

Delp, M.D., Armstrong, R.B., Godfrey, D.A., Laughlin, M.H., Ross, C.D., \& Wilkerson, M.K. (2001). Exercise increases blood flow to locomotor vestibular cardio respiratory and visual regions of the brain in miniature swine. Journal of Physiology, 533, 849859.

Dietrich, A. (2003). Functional neuroanatomy of altered states of consciousness: the transient hypofrontality hypothesis. Consciousness and Cognition, 12, 231-256. 
Drolette, E. S., Shshido, T., Pontifex, M. B., \& Hillman, C. H. (2012). Maintenance of cognitive control during and after walking in preadolescent children. Medicine and Science in Sports and Exercise, 44, 2017-2024.

Hardman, K. (2008). The situation of physical education in schools: a European perspective. Human Movement, 9(1), 5-18.

Hillman, C., Castelli, D., \& Buck, S. (2005). Aerobic fitness and neuro-cognitive function in healthy preadolescent children. Medicine and Science in Sports and Exercise, 37, 1967-1974.

Hillman, C., Pontifex, M., Raine, L., Castelli, D., Hall, E., \& Kramer, A. (2009). The effect of acute treadmill walking on cognitive control and academic achievement in preadolescent children. Neuroscience, 159, 1044-1054.

Hogervorst, E., Riedel, W., Jeukendrup, A., \& Jolles, J. (1996). Cognitive performance after strenuous physical exercise. Perceptual and Motor Skills, 83, 479-488.

Hopkins, M.E., Davis, F.C., Vantieghem, M.R., Whalen, P.J., \& Bucci, D.J. (2012). Differential effects of acute and regular physical exercise on cognition and affect. Neuroscience, 215, 59-68.

Janot, J. M. (2005). Comparing intensity monitoring methods. IDEA Fitness Journal, 2, 4.

Karvonen, J., \& Vuorimaa, T. (1988). Heart rate and exercise intensity during sports activities. Sports Medicine, 5, 303-312.

Kristjansson, A. L., Sigfúsdóttir, I. D., Allegrante, J. P., \& Helgason, A. R. (2008). Adolescent health behaviour, contentment in school, and academic achievement. American Journal of Health Behaviour, 33, 69-79. 
Lambourne, K. \& Tomporowki, P. (2010). The effect of exercise-induced arousal on cognitive task performance: A meta-regression analysis. Brain Research, 1341, 12-24.

Levitt, S. \& Gutin, B. (1971). Multiple choice reaction time and movement time during physical exertion. Research Quarterly, 42, 405-410

Lichtman, S., \& Poser, E. G. (1983). The effects of exercise on mood and cognitive functioning. Journal of Psychosomatic Research, 27, 43-52.

Lyons, M., Al-Nakeeb, Y., \& Nevill, A. (2008). The effect of moderate and high intensity fatigue on coincidence anticipation in expert and novice Gaelic games players. European Journal of Sport Science, 8, 205-216.

McMorris, T., \& Graydon, J. (2000). The effect of incremental exercise on cognitive performance. International Journal of Sport Psychology, 31, 66-81.

McMorris, T. \& Graydon, J. (1996). The effect of exercise on the decision-making performance of experienced and inexperienced soccer players. Research Quarterly for Exercise and Sport. 67, 109-114.

McMorris, T. \& Keen, P. (1994). Effect of exercise on simple reaction times of recreational athletes. Perceptual and Motor Skills, 78, 123-130.

McMorris, T., Sproule, J., Turner, A., \& Hale, B. J. (2011) Acute, intermediate intensity exercise, and speed and accuracy in working memory tasks: A metaanalytical comparison of effects. Physiology and Behavior, 102, 421-428.

Mirwald, R.L., Baxter-Jones, A., Bailey, D.A., \& Beunen, G.P. (2002). An exercise assessment of maturity from anthropometric measurements. Medicine and Science in Sports and Exercise, 34(4), 689-694. 
Parikh, T \& Stratton, G. (2011). Influence of intensity of physical activity on adiposity and cardiorespiratory fitness in 5-18 year olds. Sports Medicine 41, 477-488

Payne, V.G., \& Morrow, J.R. (1993). Exercise and VO2 max in children: a meta-analysis. Research Quarterly for Exercise and Sport, 64, 305-313.

Riddoch, C.J., Mattocks, C., Deere, K., Saunders, J., Kikby, J., Tilling, K., et al. (2007). Objective measurement if levels and patterns of physical activity. Archives of Disease in Childhood, 92, 963-969.

Ridgers N. D., Stratton G., Clark E., Fairclough S. J., \& Richardson D. J. (2006). Day-today and seasonal variability of physical activity during school recess. Preventive Medicine, 42, 372-374.

Strong, W.B., Malina, R.M., Blimkie, C.J., Daniels, S.R., Dishman, R.K., Gutin, B., et al. (2005). Evidence-based physical activity for school-age youth. Journal of Pediatrics, $146,732-737$.

Tanner, J.M. (1962). Growth at adolescence. With a general consideration of the effect of hereditary and environmental factors upon growth and maturation from birth to maturity. Oxford, UK: Blackwell Scientific.

Tomporowski, P. (2003) Cognitive and behavioural responses to acute exercise in youth: a review. Pediatric Exercise Science, 15, 348-359.

Tomporowski, P.D., Cureton, K., Armstrong, L.E., Kane, G.M., Sparling, P.B., \& MillardStafford, M. (2005). Short-term effects of aerobic exercise on executive processes and emotional reactivity. International Journal of Sports Exercise Psychology, 3, 131146. 
US Department of Health and Human Services (2013). Healthy People 2020

[online]. Available from: URL: http://www.healthypeople.gov/2020/LHI/default.aspx

[Accessed April 10, 2013].

World Health Organisation (WHO) (2011) Global recommendations on physical activity and health in 5-17 year olds. Geneva, Switzerland, World Health Organisation.

Wilkinson, G.S., \& Robertson, G.J. (2006). Wide Ranging Achievement Test (Fourth Edition). Lutz. FL: Psychological Assessment. Yerkes, R. M., \& Dodson, J. D. (1908). The relation of strength of stimulus to rapidity of habit formation. Journal of Comparative Neurology and Psychology, 18, 459-482. 
Figure captions

Figure 1: Mean standardised scores for arithmetic, spelling, reading and sentence comprehension, across the moderate exercise and rest conditions. Error bars denote +/- SEM.

Figure 2: Mean standardised scores for arithmetic, spelling, reading and sentence comprehension, across the resting, moderate exercise, and vigorous exercise conditions. Error bars denote +/- SEM. 
Figure 1

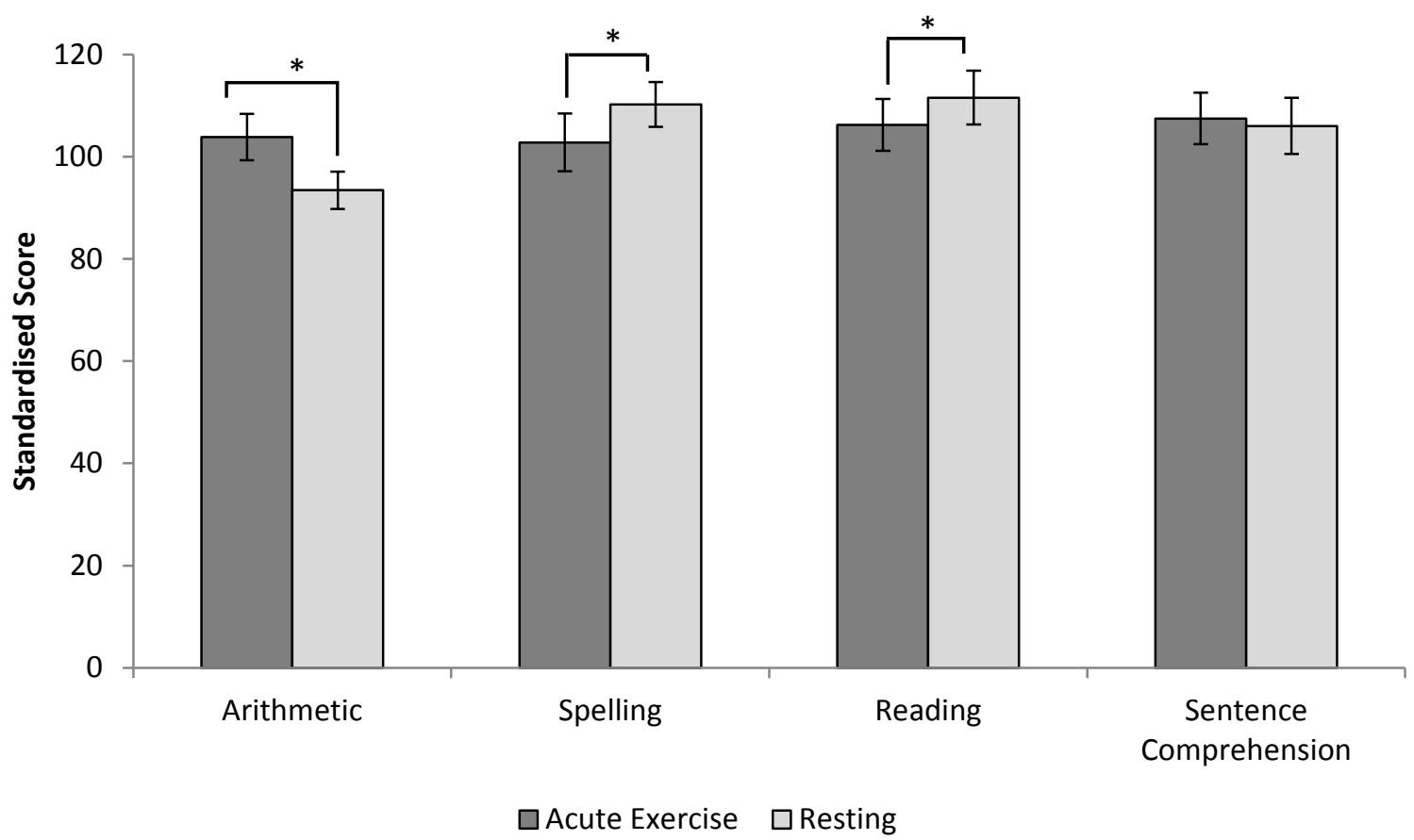

Figure 1: Mean standardised scores for arithmetic, spelling, reading and sentence comprehension, across the moderate exercise and rest conditions. Error bars denote $+/-$ SEM. 
Figure 2

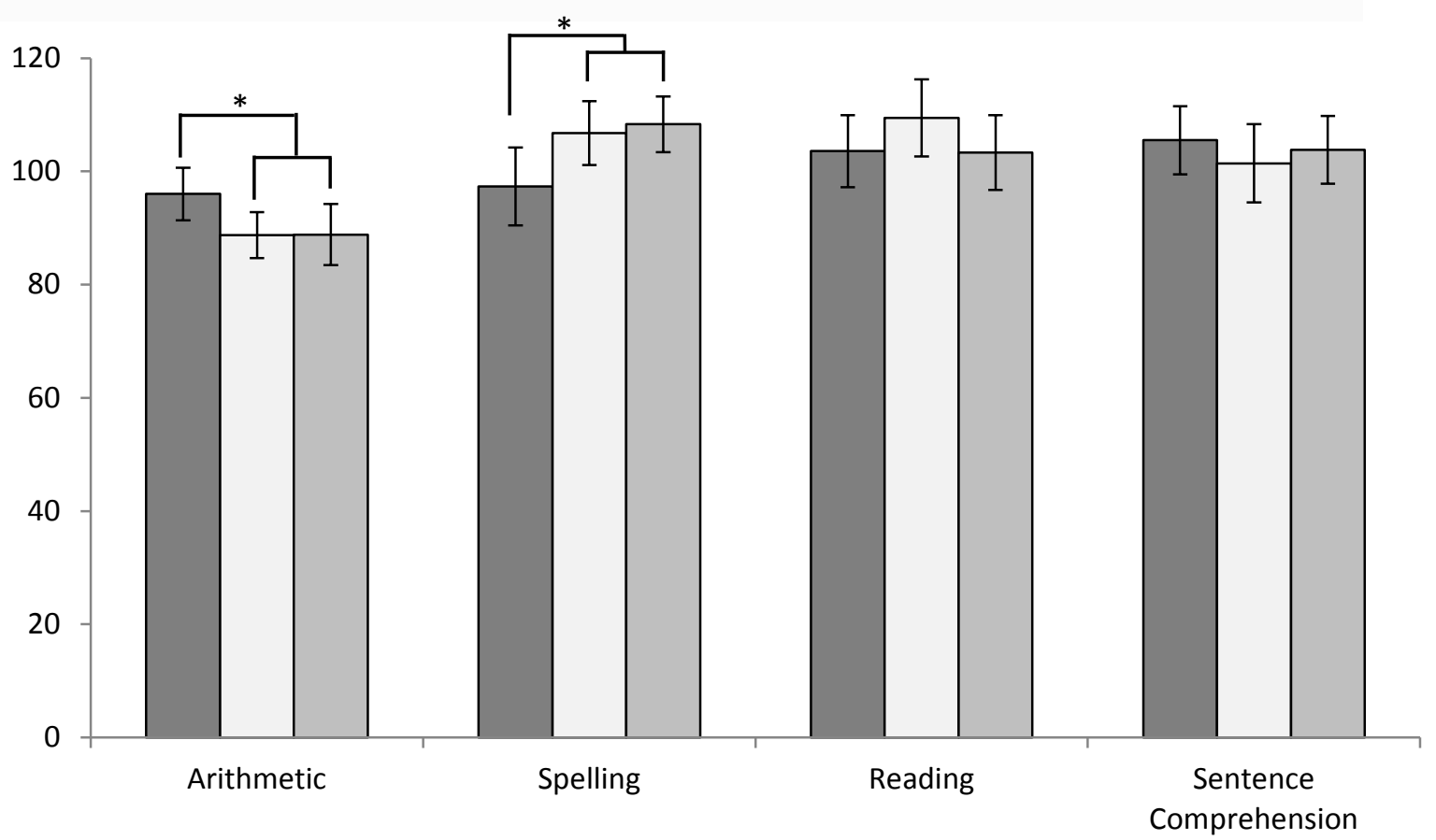

$\square$ Resting $\square$ Acute Moderate Exercise $\square$ Acute Vigorous Exercise

Figure 2: Mean standardised scores for arithmetic, spelling, reading and sentence comprehension, across the resting, moderate exercise, and vigorous exercise conditions. Error bars denote +/- SEM. 